\title{
Drospirenone-containing oral contraceptives do not affect glucose regulation and circulating corticosterone
}

\author{
${ }^{1}$ Department of Physiology, College of Health Sciences, University of Ilorin, Ilorin 240003, Nigeria \\ ${ }^{2}$ Cardiometabolic Research Unit, Department of Physiology, Afe Babalola University, Ado-Ekiti, Nigeria \\ ${ }^{3}$ Department of Physiology, College of Health Sciences, University of Ilorin, PMB 1515, Ilorin 240003, Nigeria, Phone: \\ +2348035755360, E-mail: tunjilaw@unilorin.edu.ng
}

\begin{abstract}
:
Background: Combined oral contraceptive (COC) use has been associated with an increased risk of insulin resistance (IR) and other adverse cardiovascular events, despite efforts to reduce the dosage and/or progestin type. COC containing drospirenone (DRSP) is an analog of spironolactone, hence its antimineralocorticoid and antiandrogenic characteristics have been deemed beneficial, although the benefits and/or negative outcome of its usage have not been fully elucidated. We therefore hypothesized that COC with DRSP component will not affect glucose regulation and circulating corticosterone.

Method: Ten-week-old female Wistar rats were divided into three groups: control (CON), ethinylestradiol/drospirenone COC (EE/DRSP)-treated, and ethinylestradiol/levonorgestrel COC (EE/LN)-treated rats. The treatment lasted for 8 weeks.

Results: Results showed that with the exception of lipid profiles, EE/LN but not EE/DRSP COC treatment affected body weight, glucose tolerance, plasma insulin, corticosterone, (IR), and pancreatic $\beta$-cell dysfunction. Conclusion: Taken together, the findings showed that the beneficial effect of EE/DRSP could possibly be through the DRSP component. The result also implies that COCs containing DRSP may be a better and safer means of contraception than those with LN with less cardiovascular risks.
\end{abstract}

Keywords: CVD, drospirenone, estrogen-progestin oral contraceptive, insulin resistance, glucocorticoids DOI: 10.1515/jbcpp-2018-0184

Received: October 8, 2018; Accepted: June 17, 2019

\section{Introduction}

Cardiometabolic disorder (CMD), a cluster of metabolic disorders including insulin resistance (IR), hyperglycemia, dyslipidemia, etc. [1] that occur together and place the affected individuals to higher risk of cardiovascular events [2], remains the leading cause of morbidity and mortality globally [3]. Among these cluster of metabolic disorders, IR ranks almost highest with rapid surge over the last decade, as it now affects close to 30 million people [4]. It is the primary metabolic link for the development of cardiovascular disease (CVD) [5], as it results in lower levels of uptake of glucose from the blood into target tissues, thereby causing high blood glucose level with characteristic compensatory hyperinsulinemia, which is associated with atherosclerotic CVD and mortality independent of other risk factors [6]. Despite increased efforts to prevent, treat, and control IR and its sequelae, the prevalence has not declined.

Glucocorticoids (GCs), such as cortisol in humans and corticosterone in rodents, are stress and catabolic hormones that have strong anti-inflammatory properties [7]. They are primarily responsible for mobilizing glucose to the circulation from the liver and also inhibiting the uptake and utilization of glucose. However, individuals with elevated circulating GCs present a variety of systemic manifestations that are associated with increased atherothrombotic CVD risk, such as IR, hypertension, diabetes mellitus, and dyslipidemia [8], [9].

Combined oral contraceptive (COC) is one of the most commonly prescribed birth control methods and used by millions of women around the world [10]. The adverse contribution of COC to the pathogenesis of CMD remains unresolved [11], [12] and the mechanisms still need further studies although one of it is by causing metabolic disarrangements with IR [13]. The types and doses of the estrogen component of the COC have not helped alleviate these dysfunctions [14], [15], as recent studies have shown that even COCs with low 
hormone levels (ethinylestradiol: EE), combined with/without newer generations of progestogens, may still lead to CV risk in women using the COC [16]. Attention has been drawn to COCs with formulation containing drospirenone (DRSP) [17].

DRSP is the synthetic progestin most similar to the natural progesterone, and is structurally similar to spironolactone, acting as an antagonist of aldosterone receptors with clinically recognized antimineralocorticoid activity [18]. The combination of DRSP with EE has been shown to be effective in women using COCs [19], [20], yet the negative side effects with its use have not been fully explored and studies on these have been largely inconclusive [16], [21]. The aim of this study was therefore to look at the effect of COC with DRSP component on glucose regulation and circulating corticosterone.

\section{Materials and methods}

\section{Animals}

All experimental procedures were approved by the University of Ilorin Ethical Review Committee (UIERC) with protocol number UERC/ASN/2016/364 and the procedure was done in accordance with guidelines of the National Institutes of Health (NIH) Guide for the Care and Use of Laboratory Animals; every effort was made to minimize both the number of animals used and their suffering. Ten-week-old female Wistar rats were used for the study. Rats had unrestricted access to standard rat chow and tap water. After 1 week of acclimatization of the rats to stabilize in the new environment and promote their welfare and reproducible results, the rats were randomly assigned to three groups of $\mathrm{n}=5$ each. Rats were maintained under standard environmental conditions of temperature $\left(26-28^{\circ} \mathrm{C}\right)$, relative humidity $(57-65 \%)$, and 12-h dark/light cycle.

\section{Treatment}

Control group received distilled water (vehicle; po), and COC-treated groups received (po) a combination of $1.0 \mu \mathrm{g}$ EE and $5.0 \mu \mathrm{g}$ levonorgestrel (LN) (Wyeth-Ayerst, Inc., Montreal, Canada) or a combination of $1.0 \mu \mathrm{g}$ EE and $0.3 \mathrm{mg}$ DRSP (Schering AG, Berlin, Germany), respectively, and adjusted for body weight. The treatments were daily and lasted for 8 weeks.

\section{Sample preparation}

At the end of treatment, the rats were anesthetized with pentobarbital sodium (50 mg/ kg, ip). Blood was collected by cardiac puncture into EDTA-coated and heparinized bottles, accordingly. Blood collected in heparinized bottles was centrifuged at $3000 \mathrm{rpm}$ for $5 \mathrm{~min}$. Plasma was stored frozen until needed for biochemical assay. Plasma and blood viscosity were estimated according to the Vand formula [22] as adapted in our laboratory [23].

\section{Oral glucose tolerance test (OGTT), IR, and pancreatic $\beta$-cell function}

Glucose challenge test was performed $48 \mathrm{~h}$ before the end of the experiment. The rats were kept in fasting condition for $12 \mathrm{~h}$ overnight. Blood sample was collected by the tail-cut method before glucose load, and blood glucose level was assessed by a glucometer (ONETOUCH ${ }^{\circledR}$-LifeScan, Inc., Milpitas, CA, USA); it was denoted as the 0 th time point. Glucose ( $2 \mathrm{~g} / \mathrm{kg}$ bw) was administered orally (po), and blood glucose was assessed subsequently after $30,60,90$, and $120 \mathrm{~min}$. Glucose tolerance was expressed as a function of the area under the OGTT curve (AUC) as previously described [24] and post-load glucose responses. IR was determined using the homeostasis model assessment for insulin resistance (HOMA-IR $=$ fasting glucose $(\mathrm{mmol} / \mathrm{L}) \times$ fasting insulin $(\mu \mathrm{U} / \mathrm{L}) / 22.5)$ whereas HOMA- $\beta(20 \times$ fasting insulin $(\mu \mathrm{U} / \mathrm{L}) /$ fasting glucose -3.5$)$ and 1 -h post-load glucose levels were used to determine pancreatic $\beta$-cell function. Elevated1-h post-load glucose level is a predictor of IR, pancreatic $\beta$-cell function, atherosclerotic CVD, and renal dysfunction [25], [26]. 


\section{Biochemical assays}

The plasma levels of corticosterone were estimated using ELISA kits (Elabscience Biotechnology Co., Ltd., Wuhan, China). Insulin was also determined using an ELISA kit (Ray Biotechnology, Cruz, Canada). The biological markers of dyslipidemia including total cholesterol (TC), triglyceride (TG), high-density lipoproteincholesterol (HDL-C) were measured by standardized enzymatic colorimetric methods using reagents obtained from Randox Laboratory Ltd. (Antrim, UK). The atherogenic indices (TC/HDL-C and TG/HDL-C ratios) were also estimated.

\section{Statistical analysis}

All experimental data were expressed as means \pm SEM. Statistical significance for the measured variables was determined by one-way analysis of variance (ANOVA) for the comparison of the mean values of variables among the groups. Bonferroni's test was used to identify the significance of pairwise comparison of mean values among the groups. Statistically significant differences were accepted at $p<0.05$. Statistical group analysis was performed with the SPSS statistical software.

\section{Results}

\section{Effects of EE/LN vs. EE/DRSP treatments on body weight, food, and water intake}

The initial body weight was comparable among all the experimental groups. However, for the EE/LN group, the final body weight was lower. Food and water intake was not significantly affected (Table 1).

Table 1: Effect of combined oral contraceptives containing levonorgestrel and drospirenone on body weight, food and water intake, fasting glycemia, and insulinemia in rats.

\begin{tabular}{lrrr}
\hline & CON & EE/LN & EE/DRSP \\
\hline Body weight, & & & \\
$\quad$ Initial & $117.8 \pm 10.0$ & $117.7 \pm 7.0$ & $117.1 \pm 8.0$ \\
$\quad$ Final & $162.9 \pm 10.9$ & $131.3 \pm 5.4^{\mathrm{a}}$ & $162.3 \pm 6.4^{\mathrm{b}}$ \\
Food intake, g/kg/day & $72.8 \pm 2.9$ & $70.9 \pm 6.7$ & $78.0 \pm 7.5$ \\
Water intake, mL/kg/day & $192.4 \pm 10.0$ & $196.4 \pm 7.0$ & $207.3 \pm 6.6$ \\
Fasting glycemia, mmol/L & $3.5 \pm 0.1$ & $3.4 \pm 0.2$ & $3.5 \pm 0.1$ \\
Fasting insulinemia, & $20.0 \pm 1.1$ & $31.1 \pm 2.2^{\mathrm{a}}$ & $20.2 \pm 1.5$ \\
$\mu \mathrm{IU} / \mathrm{mL}$ & & &
\end{tabular}

\footnotetext{
Data are expressed as mean \pm SEM of five rats per group. Data were analyzed by one-way ANOVA followed by Bonferroni post hoc test $\left({ }^{a} p<0.05\right.$ vs. CON; ${ }^{b} p<0.05$ vs. EE/LN).
}

\section{Effects of EE/LN vs. EE/DRSP treatments on glucose regulation and IR}

Fasting glycemia was comparable in all the experimental groups (Table 1). EE/LN treatment led to significant increase in 1 and 1.5-h post-load glycemia changes, respectively, compared with CON, whereas EE/DRSP treatment at 1 and $1.5 \mathrm{~h}$, respectively, did not change significantly compared with CON and EE/LN, respectively (Figure 1B,C). However, 2-h post-load glycemia was not affected in all the experimental groups (Figure 1D). 
A

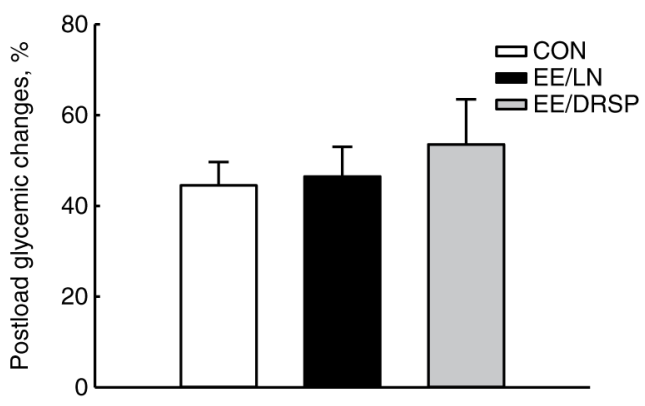

C

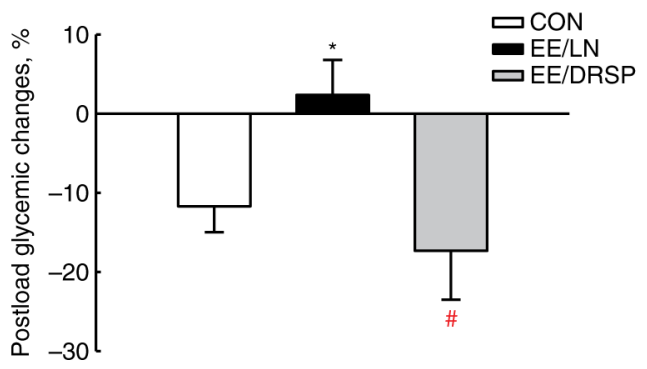

B

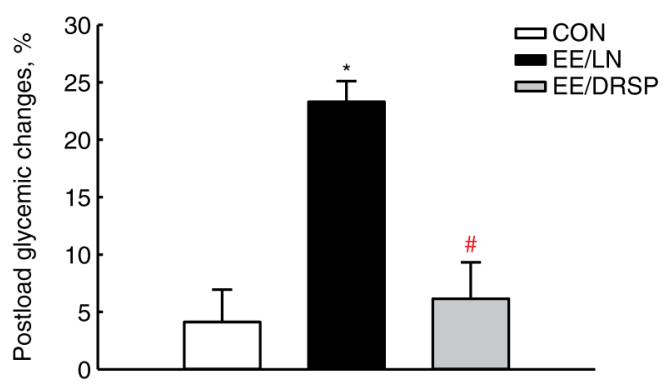

D

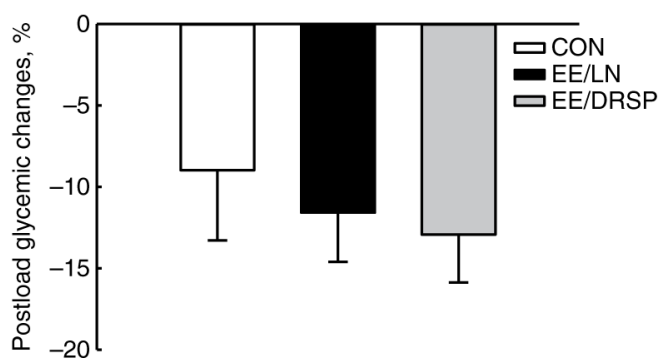

Figure 1: COCs containing drospirenone do not cause impaired glucose tolerance.

Effect of COCs containing levonorgestrel (EE/LN) or drospirenone (EE/DRSP) treatments on percentage post-load glycemic changes at 30, 60, 90, and $120 \mathrm{~min}$ (A-D, respectively) in female Wistar rats. EE/LN but not EE/DRSP caused impaired glucose tolerance and elevated 1- and 1.5-h post-load glucose response, respectively. Data were analyzed by one-way ANOVA followed by Bonferroni post hoc test. Values are expressed as mean \pm SEM of five rats per group $\left({ }^{\mathrm{a}} \mathrm{p}<\right.$ 0.05 vs. $\mathrm{CON} ;{ }^{\mathrm{b}} \mathrm{p}<0.05$ vs. EE/LN).

In addition, the AUC of OGTT was higher in the EE/LN-treated group than in CON but reduced in the EE/DRSP group compared with EE/LN (Figure 2A). EE/LN treatment led to an increase in fasting plasma insulin (Table 1) HOMA-IR (Figure 2B), and HOMA- $\beta$ (Figure 2C) compared with the CON group, which were all not affected by EE/DRSP treatment compared with EE/LN treatment.

A
B

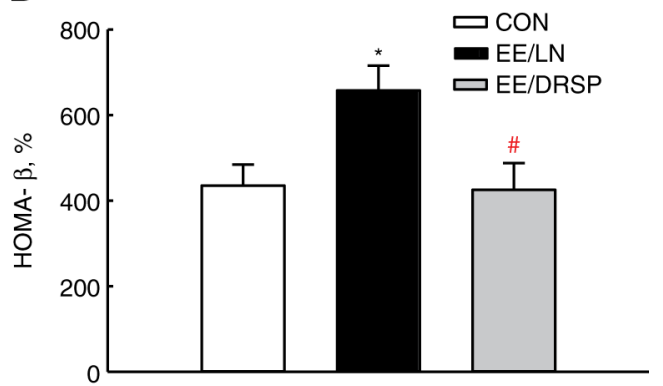

C

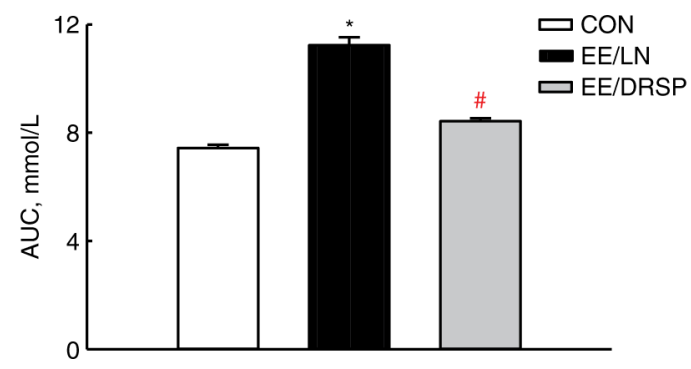

Figure 2: COCs containing levonorgestrel and drospirenone both affected atherogenic dyslipidemia.

Effect of COCs containing levonorgestrel (EE/LN) or drospirenone (EE/DRSP) treatments on (A) insulin resistance, (B) pancreatic $\beta$-cell function, and (C) area under the curve of oral glucose tolerance test in female Wistar rats. EE/LN but not EE/DRSP caused insulin resistance, impaired pancreatic $\beta$-cell function and elevated area under the curve of oral glucose tolerance test. Data were analyzed by one-way ANOVA followed by Bonferroni post hoc test. Values are expressed as mean \pm SEM of five rats per group $\left({ }^{a} \mathrm{p}<0.05 \mathrm{vs}\right.$. CON; ${ }^{b} \mathrm{p}<0.05 \mathrm{vs}$. EE/LN). 


\section{Effects of EE/LN vs. EE/DRSP treatments on atherogenic lipids and blood viscosity}

The plasma HDL cholesterol levels were significantly lowered in all the treated groups compared with the control. EE/LN treatment led to significant increases in biological markers of dyslipidemia such as plasma TG, TC and LDL cholesterol levels compared with the control and EE/DRSP-treated groups (Table 2). EE/LN and EE/DRSP treatments led to significant increase in the atherogenic indices (TG/HDL-C and TC/HDL-C) compared with the CON group (Figure 3A,B). Plasma viscosity was comparable in all the groups (Figure 4A). However, blood viscosity (Figure 4B) and percentage hematocrit (Figure 4C) levels were significantly raised in the EE/LN-treated group compared with the CON and EE/DRSP group, respectively.

Table 2: Effect of combined oral contraceptives containing levonorgestrel and drospirenone on triglyceride (TG), total cholesterol (TC), high-density lipoprotein cholesterol (HDL-C), and low-density lipoprotein (LDL-C) in rats.

\begin{tabular}{lrrr}
\hline & CON & EE/LN & EE/DRSP \\
\hline TG, mg/dL & $61.7 \pm 6.6$ & $138.2 \pm 1.4^{\mathrm{a}}$ & $120.1 \pm 1.9^{\mathrm{a}, \mathrm{b}}$ \\
TC, mg/dL & $46.5 \pm 2.6$ & $91.9 \pm 3.4^{\mathrm{a}}$ & $71.9 \pm 3.5^{\mathrm{a}, \mathrm{b}}$ \\
HDL-C, mg/dL & $70.6 \pm 14.2$ & $39.5 \pm 5.6^{\mathrm{a}}$ & $35.3 \pm 3.8^{\mathrm{a}}$ \\
LDL-C, mg/dL & $2.7 \pm 1.9$ & $30.9 \pm 3.4^{\mathrm{a}}$ & $14.9 \pm 3.7^{\mathrm{a}, \mathrm{b}}$ \\
\hline
\end{tabular}

Data are expressed as mean \pm SEM of five rats per group. Data were analyzed by one-way ANOVA followed by Bonferroni post hoc test $\left({ }^{\mathrm{a}} \mathrm{p}<0.05\right.$ vs. CON; ${ }^{\mathrm{b}} \mathrm{p}<0.05$ vs. EE/LN).

A

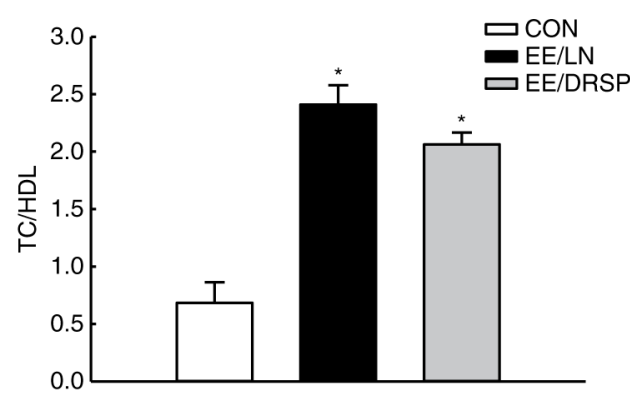

B

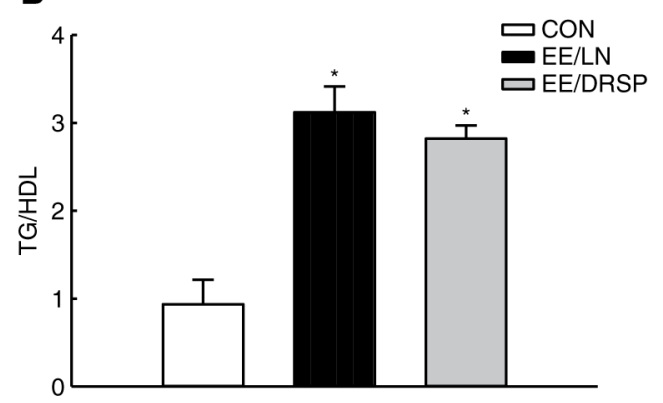

Figure 3: COCs containing drospirenone do not cause insulin resistance.

Effect of COCs containing levonorgestrel (EE/LN) or drospirenone (EE/DRSP) treatments on atherogenic indices (A, B) in female Wistar rats. EE/LN and EE/DRSP caused elevated TG/HDL-C and TC/HDL-C ratios. Data were analyzed by one-way ANOVA followed by Bonferroni post hoc test. Values are expressed as mean \pm SEM of five rats per group $\left({ }^{a} \mathrm{p}<\right.$ 0.05 vs. $\mathrm{CON} ;{ }^{b} \mathrm{p}<0.05$ vs. $\left.\mathrm{EE} / \mathrm{LN}\right)$.

A

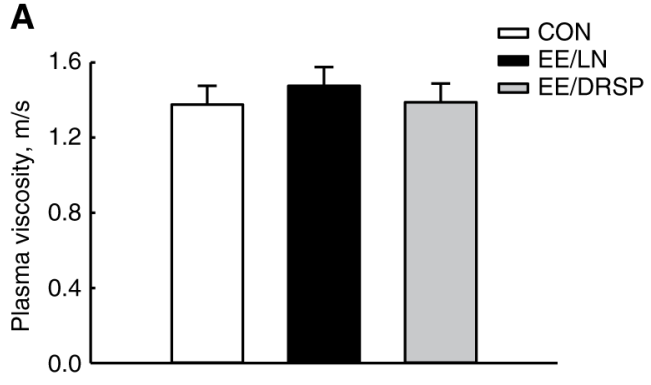

B

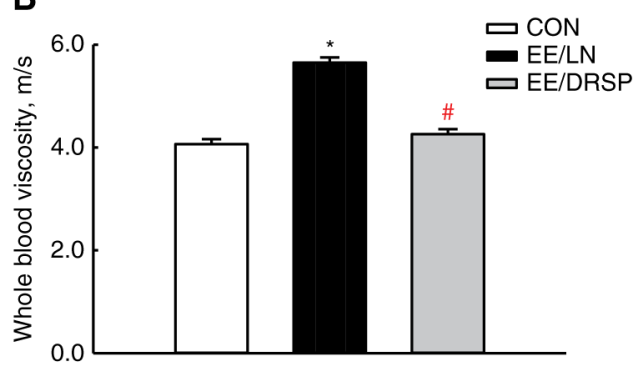

\section{C}

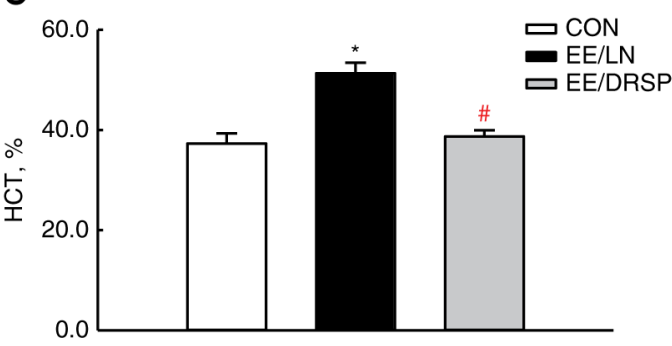


Figure 4: COCs containing levonorgestrel altered the whole blood viscosity. Effect of COCs containing levonorgestrel (EE/LN) or drospirenone (EE/DRSP) treatments on (A) plasma viscosity, (B) whole blood viscosity, and (C) hematocrit level in female Wistar rats. EE/LN but not EE/DRSP increased whole blood viscosity and hematocrit levels. Data were analyzed by one-way ANOVA followed by Bonferroni post hoc test. Values are expressed as mean \pm SEM of five rats per group ( ${ }^{\mathrm{a}} \mathrm{p}<0.05 \mathrm{vs}$. CON; ${ }^{\mathrm{b}} \mathrm{p}<0.05 \mathrm{vs}$. EE/LN).

\section{Effects of EE/LN vs. EE/DRSP treatments on circulating GCs}

EE/LN treatment led to significant increase in circulating corticosterone level compared with the CON and $\mathrm{EE} / \mathrm{LN}$-treated groups. However, there was no change in the level of corticosterone level in the EE/DRSP group compared with the CON group (Figure 5).

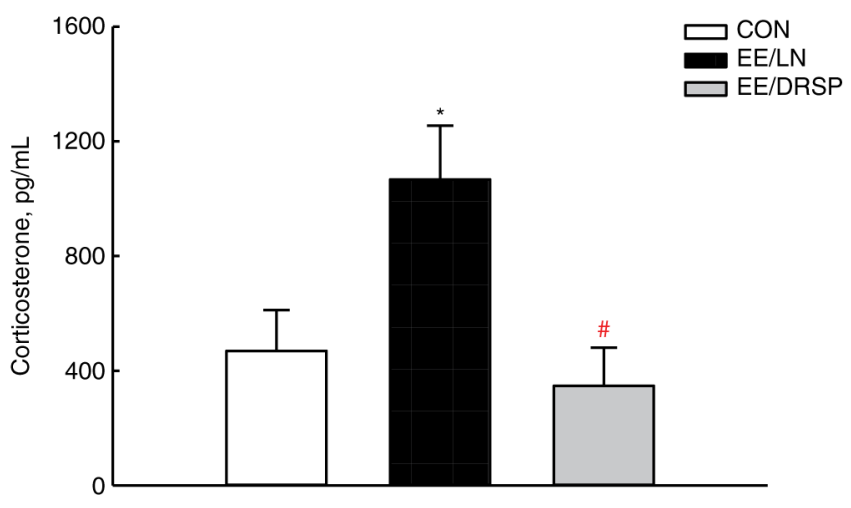

Figure 5: Effect of COCs containing levonorgestrel (EE/LN) or drospirenone (EE/DRSP) treatments on circulating corticosterone in female Wistar rats.

EE/LN but not EE/DRSP increased circulating corticosterone. Data were analyzed by one-way ANOVA followed by Bonferroni post hoc test. Values are expressed as mean \pm SEM of five rats per group ( ${ }^{\mathrm{p}} \mathrm{p}<0.05 \mathrm{vs}$. CON; ${ }^{\mathrm{b}} \mathrm{p}<0.05 \mathrm{vs}$. EE/LN).

\section{Discussion}

The results of the present study demonstrate that EE/LN caused IR, which was accompanied by pancreatic $\beta$-cell dysfunction, glucose deregulation, dyslipidemia, as well as increased blood viscosity and circulating corticosterone. The alterations in these parameters were however not noticed in the EE/DRSP-treated group.

IR has been shown to be the hallmark of CVD risk factors [27]. It is associated with decreased glucose uptake in insulin-sensitive tissues such as the skeletal muscle and liver. IR is rapidly becoming one of the major public health challenges, signifying an enormous global economic burden [28], particularly when accompanied by elevated fasting and 1-h post-load glycemia [29], [30]. Elevated 1-h post-load glycemia has been shown to be a reliable, independent determinant of impaired pancreatic $\beta$-cell function and IR, which are accompanied by increased atherosclerotic CVD even in individuals otherwise regarded as having normal glucose tolerance [31]. Hence, factors or intervention that could promote or prevent the development of IR, impaired 1-h post-load glycemia, and its associated atherogenic dyslipidemia will be of great clinical and public health importance, particularly in COC users. Our study showed evidence that EE/DRSP treatment prevented IR and elevated 1-h post load. This may be due to the DRSP component, which is known to exhibit a potent anti-aldosterone activity as it blocks the mineralocorticoid receptor (MR) that can bring about IR and other CVD risk factors if activated [17]. More so, it has been demonstrated in our laboratory that EE/LN can activate MR [12], further proving the beneficial use of EE/DRSP, possibly due to the DRSP component. The findings that 2-h post-load glycemia and fasting glycemia (Figure 1D) were comparable in all the experimental groups do not undermine the effectiveness of EE/DRSP in glucose homeostasis, as elevated 1-h post-load glycemia has been shown to be a stronger independent predictor of pancreatic $\beta$-cell dysfunction and cardiometabolic complications than 2-h post-load glycemia [32]. In addition, the anti-aldosterone effect of DRSP can reduce estrogen-related sodium and water retention, which regulates sodium and water balance. This may translate into weight benefits as noticed in our result [33]. Interestingly, there was reduction in body weight in the EE/LN-treated rats.

Dyslipidemia, characterized by elevated TG, TC, TG/HDL-C, and TC/HDL-C, was observed in both EE/LN- and EE/DRSP-treated rats. The dyslipidemia observed in the EE/LN-treated rats may be due to the observed hyperinsulinemia and IR that was noticed in the group [33]. Studies have demonstrated that atherogenic lipid ratios (TG/HDL-C and TC/HDL-C) are useful markers in identifying individuals at risk for devel- 
oping CVD [34]. Although not significant, the ratios were reduced in the EE/DRSP-treated group compared with EE/LN-treated rats. This is in consonance with previous reports [35], [36]. More so, previous reports have shown the beneficial effect of EE/DRSP on some cardiovascular risk factors such as hypertension yet little or no impact on other cardiometabolic profiles such as dyslipidemia [37], [38]. Hence, EE/DRSP treatment had lesser risk on lipid metabolism. However, it should be noted that the variability might be due to the combined actions of EE and DRSP, as the action of DRSP has been shown to be cardioprotective [36], [39]. Altered blood rheology has been linked to the development of atherothrombotic complications, and increased systemic inflammation seems to be an important clinical correlate [40], [41]. Studies have shown that altered levels of blood rheological markers, which affect blood flow in both macro-and micro-circulations, are associated with incident atherothrombotic CVD. These markers include blood viscosity and its major determinants, namely hematocrit and plasma viscosity [40]. Blood viscosity and hematocrit level were elevated in the EE/LN-treated rats, but were however not affected in the EE/DRSP-treated animals, compared with CON, further confirming the effectiveness and superiority of EE/DRSP COC over EE/LN COC. More so, blood viscosity, which is an index of blood rheology, is positively correlated with blood pressure and has been related to future cardiovascular events, as was shown in a previous report [42].

GCs have been reported to cause impaired insulin action/dysmetabolism [43], [44]. The elevated GC in the EE/LN group is consistent with previous studies in our laboratory [11], [12]. An interesting finding in this work is the anti-GC activity of EE/DRSP for the first time. This also is possibly due to the DRSP component. This is contrary to a previous report that DRSP lacks anti-GC or even GC activity [45]. Although aldosterone was not estimated, which is one of the limitations of this work, it has been shown that both corticosterone and aldosterone have equal affinity to MR [46].

\section{Conclusion}

The present study demonstrates that DRSP-containing COC seems not to affect glucose regulation, IR, and circulating corticosterone but did affect lipid metabolism, though, to a lesser extent compared with EE/LN. The beneficial effects of EE/DRSP could have largely been through the DRSP component of the COC. Taken together, the findings demonstrated that COCs containing DRSP may be a better and safer means of contraception than that those containing LN, with lesser cardiovascular risks. Since the goal of this research is limited to the parameters estimated, there is great need to further our knowledge of DRSP-containing COCs with studies that analyze various types of other COC formulations in order to reach any conclusion on the real benefit of this COC on vascular pathophysiology.

\section{Acknowledgments}

The authors appreciate the technical support of the HOPE Cardiometabolic Research Team, Ilorin, Nigeria.

Author contributions: All authors have accepted responsibility for the entire content of this submitted manuscript and approved its submission.

Research funding: None declared.

Employment or leadership: None declared.

Honorarium: None declared.

Competing interests: Authors state no potential conflict of interest.

Ethical approval: Research involving animals complies with all relevant national regulations and institutional policies for the care and use of animals.

\section{References}

[1] Cameron A. The metabolic syndrome: validity and utility of clinical definitions for cardiovascular disease and diabetes risk prediction. Maturitas 2010;65:117-21. 
[2] Wilson PW, D'agostino RB, Parise H, Sulivan L, Meigs ]B. Metabolic syndrome as a precursor of cardiovascular disease and type 2 diabetes mellitus. Circulation 2005;112:3066-72.

[3] Wang ], Ruotsalainen S, Moilanen L, Lepisto P, Laakso M, Kuusisto ]. The metabolic syndrome predicts cardiovascular mortality: a 13-year follow-up study in elderly non-diabetic Finns. Eur Heart ] 2007;28:857-64.

[4] Centers for Disease Control and Prevention (CDC). National diabetes statistics report [Internet]. Atlanta, GA: Centers for Disease Control and Prevention, 2014.

[5] Sasso FC, Carbonara O, Nasti R, Campana B, Marfella R, Torella M, et al. Clucose metabolism and coronary heart disease in patients with normal glucose tolerance. J Am Med Assoc 2004;291:1857-63.

[6] Abdul-Chani MA, Tripathy D, DeFronzo RA. Contributions of beta-cell dysfunction and insulin resistance to the pathogenesis of impaired glucose tolerance and impaired fasting glucose. Diabetes Care 2006;29:1130-9.

[7] Rhen T, Cidlowski JA. Anti-inflammatory action of glucocorticoids - new mechanisms for old drugs. N Engl ] Med 2005;353:1711-23.

[8] Dekkers OM, Horvath-Puho E, Jorgensen JO, Cannegieter SC, Ehrenstein V, Vandenbroucke JP, et al. Multisystem morbidity and mortality in Cushing's syndrome: a cohort study. ] Clin Endocrinol Metab 2013;98:2277-84.

[9] Coelho MC, Santos CV, Vieira Neto L, Gadelha MR. Adverse effects of glucocorticoids: coagulopathy. Eur ] Endocrinol 2015;173:M11-21.

[10] Shufelt CL, Merz CN. Contraceptive hormone use and cardiovascular disease. ] Am Coll Cardiol 2009;53:221-31.

[11] Olatunji LA, Omolekulo TE, Usman TO, Kim I. Improvement of oral contraceptive-induced glucose dysregulation and dyslipidemia by valproic acid is independent of circulating corticosterone. Arch Physiol Biochem 2016;122:123-9.

[12] Adeyanju OA, Michael OS, Soladoye AO, Olatunji LA. Blockade of mineralocorticoid receptor ameliorates oral contraceptive-induced insulin resistance by suppressing elevated uric acid and glycogen synthase kinase-3 instead of circulating mineralocorticoid. Arch Physiol Biochem 2018. Available at: https://doi.org/10.1080/13813455.2018.1509220 (ahead of print). Accessed January 2019.

[13] Baillargeon JP, McClish DK, Essah PA, Nestler]E. Association between the current use of low-dose oral contraceptives and cardiovascular arterial disease: a meta-analysis. J Clin Endocrinol Metab 2014;90:3863-70.

[14] Practice Committee of the American Society for Reproductive Medicine. Hormonal contraception: recent advances and controversies. Fertil Steril 2008;90:103-13.

[15] Centers for Disease Control and Prevention. US medical eligibility criteria for contraceptive use. Adapted from the World Health Organization medical eligibility criteria for contraceptive use. 4th edition. MMWR Early Release 2010;59:1-6.

[16] Giribela CR, Melo NR, Silva RC, Hong VM, Guerra CM, Baracat EC, et al. A combined oral contraceptive containing drospirenone changes neither endothelial function nor hemodynamic parameters in healthy young women: a prospective clinical trial. Contraception 2012;86:35-41.

[17] Palacios S, Foidart JM, Genazzani AR. Advances in hormone replacement therapy with drospirenone, a unique progestogen with aldosterone receptor antagonism. Maturitas 2006;55:297-307.

[18] Oelkers W. Drospirenone, a progestogen with antimineralocorticoid properties: a short review. Mol Cell Endocrinol 2004;217:255-61.

[19] Guido M, Romualdi D, Ciuliani M, Suriano R, Selvaggi L, Apa R, et al. Drospirenone for the treatment of hirsute women with polycystic ovary syndrome: a clinical, endocrinological, metabolic pilot study. ] Clin Endocrinol Metab 2004;89:2817-23.

[20] Pehlivanov B, Mitkov M. Efficacy of an oral contraceptive containing drospirenone in the treatment of women with polycystic ovary syndrome. Eur ] Contracept Reprod Health Care 2007;12:30-5.

[21] Seeger ]D, Loughlin ], Eng PM, Clifford CR, Cutone ], Walker AM. Risk of thromboembolism in women taking ethinylestradiol/drospirenone and other oral contraceptives. Obstet Cynecol 2007;110:587-93.

[22] Cordon R]. Viscosity considerations in the determination of regional circulatory adjustments to anemia. Circ Res 1972;31:805-12.

[23] Olatunji LA, Oyeyipo IP, Usman TO. Effect of a high-fructose diet on glucose tolerance, plasma lipid and hemorhaeological parameters during oral contraceptive administration in female rats. Clin Hemorheol Microcirc 2013;54:23-31.

[24] Olatunji LA, Michael OS, Adewumi FO, Aiyegboyin I], Olatunji VA. Combined estrogen-progestogen but not progestogen-only oral contraceptive alters glucose tolerance and plasma lipid profile in female rats. Pathophysiology 2012;19:29-34

[25] Succurro E, Arturi F, Lugara M, Grembiale A, Fiorentino TV, Caruso V, et al. One hour postload plasma glucose levels are associated with kidney dysfunction. Clin ] Am Soc Nephrol 2010;5:1922-7.

[26] Bianchi C, Miccoli R, Trombetta M, Giorgino F, Frontoni S, Faloia E, et al. Elevated 1-hour postload plasma glucose levels identify subjects with normal glucose tolerance but impaired $\beta$-cell function, insulin resistance, and worse cardiovascular risk profile: the GENFIEV study. J Clin Endocrinol Metab 2013;98:2100-5.

[27] DeFronzo RA. Insulin resistance, lipotoxicity, type 2 diabetes and atherosclerosis: the missing links. The Claude Bernard Lecture 2009. Diabetologia 2010;53:1270-87.

[28] Nguyen QM, Srinivasan SR, Xu ]H, Chen W, Berenson CS. Fasting plasma glucose levels within the normoglycemic range in childhood as a predictor of prediabetes and type 2 diabetes in adulthood: the Bogalusa heart study. Arch Pediatr Adolesc Med 2010;164:124-8.

[29] Wang H, Shara NM, Calhoun D, Umans ]G, Lee ET, Howard BV. Incidence rates and predictors of diabetes in those with prediabetes: the strong heart study. Diab/Metab Res Rev 2010;26:378-85.

[30] Abdul-Chani MA, Williams K, DeFronzo RA, Stern M. What is the best predictor of future type 2 diabetes? Diabetes Care 2007;30:1544-8.

[31] Fonseca VA. Early identification and treatment of insulin resistance: impact on subsequent prediabetes and type 2 diabetes. Clin Cornerstone 2010;8:S7-18.

[32] White WB, Hanes V, Chauhan V, Pitt B. Effects of a new hormone therapy, drospirenone and 17-betaestradiol, in postmenopausal women with hypertension. Hypertension 2006;48:246-53

[33] Belcastro V, D’Egidio C, Striano P, Verrotti A. Metabolic and endocrine effects of valproic acid chronic treatment. Epilepsy Res 2013;107:18.

[34] Musso C, Graffigna M, Soutelo ], Honfi M, Ledesma L, Miksztowicz V, et al. Cardiometabolic risk factors as apolipoprotein B, triglyceride/HDL-cholesterol ratio and C-reactive protein, in adolescents with and without obesity: cross sectional study in middle class suburban children. Pediatr Diab 2011;12:229-34. 
[35] Shulman LP. A review of drospirenone for safety and tolerability and effects on endometrial safety and lipid parameters contrasted with medroxyprogesterone acetate, levonorgestrel, and micronized progesterone. J Women's Health 2006;15:584-90.

[36] Foidart JM, Faustmann T. Advances in hormone replacement therapy: weight benefits of drospirenone, a 17-alphaspirolactone-derived progestogen. Gynecol Endocrinol 2007;23:692-9.

[37] Nisenbaum MC, Melo NR, Giribela CR, de Morais TL, Guerra GM, de Angelis K, et al. Effects of a contraceptive containing drospirenone and ethinyl estradiol on blood pressure and autonomic tone: a prospective controlled clinical trial. Eur] Obstet Cynecol Reprod Biol 2014;175:62-6.

[38] White WB, Pitt B, Preston RA, Hanes V. Antihypertensive effects of drospirenone with 17-beta-estradiol, a novel hormone treatment in postmenopausal woman with stage 1 hypertension. Circulation 2005;112:1979-80.

[39] Moro F, Morciano A, Tropea A, Sagnella F, Palla C, Scarinci E, et al. Effects of drospirenone-ethinylestradiol and/or metformin on CD4 + CD28null T lymphocytes frequency in women with hyperinsulinemia having polycystic ovary syndrome: a randomized clinical trial. Reprod Sci 2013;20:1508-17.

[40] Woodward M, Rumley A, Tunstall-pedoe H, Lowe GD. Associations of blood rheology and interleukin-6 with cardiovascular risk factors and prevalent cardiovascular disease. Br] Haematol 1999;104:246-57.

[41] Tzoulaki I, Murray CD, Lee A], Rumley A, Lowe CD, Fowkes FC. Relative value of inflammatory, hemostatic, and rheological factors for incident myocardial infarction and stroke: the Edinburgh artery study. Circulation 2007;115:2119-27.

[42] Koenig W, Sund M, Filipiak B, Döring A, Löwel H, Ernst E. Plasma viscosity and the risk of coronary heart disease. Arterioscler Thromb Vasc Biol 1998;18:768-72.

[43] Schacke H, Docke WD, Asadullah K. Mechanisms involved in the side effects of glucocorticoids. Pharmacol Ther 2002;96:23-43.

[44] Fransson L, Franzén S, Rosengren V, Wolbert P, Sjöholm Å, Ortsäter H. $\beta$-cell adaptation in a mouse model of glucocorticoid-induced metabolic syndrome. J Endocrinol 2013;18:JOE-13.

[45] Preston RA, White WB, Pitt B, Bakris G, Norris PM, Hanes V. Effects of drospirenone/17-beta estradiol on blood pressure and potassium balance in hypertensive postmenopausal women. AJH 2005;18:797-804.

[46] Seckl JR. 11 $\beta$-hydroxysteroid dehydrogenase isoforms and their implications for blood pressure regulation. Eur] Clin Invest 1993;23:589601. 